\title{
Evaluation of Instantaneous Reliability Measures for a Gradual Deteriorating System
}

\author{
${ }^{1}$ Tijjani A. Waziri, ${ }^{2}$ Bashir M. Yakasai, and ${ }^{3}$ Ibrahim Yusuf \\ ${ }^{1}$ School of Continuing Education, Bayero University, Kano, Nigeria, ti j janiw@gmai1.com \\ ${ }^{2}$ Department of Mathematical Sciences, Bayero University, Kano, Nigeria, yakasaibashir911@gmai1. com \\ ${ }^{3}$ Department of Mathematical Sciences, Bayero University, Kano, Nigeria, i yusuf. mth@buk. edu. ng
}

\begin{abstract}
This paper studies various reliability measures. Reliability measures are quantities used for analyzing reliability and maintenance models. Sometimes when the maintenance cost rate is minimized, the system reliability measures are so low that they are not acceptable in practice. The following instantaneous reliability measures are considered in this paper: instantaneous failure rate, instantaneous repair rate, instantaneous maintainability, instantaneous reliability and instantaneous availability. The explicit expression and numerical results for the instantaneous reliability measures are shown. Also, their relationships are shown.
\end{abstract}

Keywords: Reliability, Gradual, Deterioration, Instantaneous, System

2000 MSC No: 90B25.

\section{Introduction}

Maintenance involves preventive (planned) and unplanned actions carried out to retain a system or restore it to an acceptable operating condition. Optimal maintenance policies aim to provide optimum system reliability and safety performance at the lowest possible maintenance costs. Proper maintenance techniques have been emphasized due to increased safety and reliability requirements of systems, increased complexity, and rising costs of material and labor. One important research area in reliability engineering is the study of various maintenance policies in order to improve system reliability, to prevent the occurrence of system failure, and to reduce maintenance costs. Reliability theory has grown out of the valuable experiences from many defects of military systems in World War II and with the development of modern technology. For the purpose of making good products with high quality and designing highly reliable systems, the importance of reliability has been increasing greatly with the innovation of recent technology. The theory has been actually applied to not only industrial, mechanical, and electronic engineering but also to computer, information, and communication engineering. Many researchers have investigated statistically and stochastically complex phenomena of real systems to improve their reliability. During operation the strengths of systems are gradually deteriorated, until some point of deterioration failure, or other types of failures. To proper deal with repairable systems that deteriorates, we need to understand the characteristics and the behavior of the system. And this will be done by investigating the properties of reliability measures. Reliability measures are quantities for analyzing reliability and maintenance models. It is important to note that, minimizing the system maintenance cost rate for a particular system may not result in optimal system reliability measures. Sometimes when the maintenance cost rate is minimized, the system reliability measures are so low that they are not acceptable in practice. The instantaneous reliability measures that are considered in this paper are: instantaneous failure rate, instantaneous repair rate, instantaneous maintainability, instantaneous reliability and instantaneous availability. 
There is an extensive literature on reliability measures. See Barlow and Proschan [2], Elsayed [3], Nakagawa[4], Pham [5] and Wang and Pham [8]. Also, many researchers have studied availability for various types of systems. For example, see Yusuf and Hussain [9], Yusuf and Yusuf [10], Yusuf et al. [11], Sun et al. [7]. Moreover, Suleiman et al. [6] and Aliyu et al. [1], analyzed the characteristics of reliability and availability of series - parallel subsystems in different configurations.

\section{Notation and Description of the System}

\subsection{Description of the System:}

Consider a system, which gradually deteriorates with time and usage. The system is subject to a particular failure, which is rectify by minimal repair.

\subsection{Notations:}

$r(t)$ : Instantaneous failure rate of the system at time $\mathrm{t}$

$h(t)$ : Instantaneous repair rate of the system at time $t$

$F(t)$ : Failure distribution of the system at time $t$

$\mathrm{f}(\mathrm{t})$ : Time to failure (pdf) the system at time $\mathrm{t}$

$\mathrm{g}(\mathrm{t})$ : Time to repair $(\mathrm{pdf})$ the system at time $\mathrm{t}$

$I R(t)$ : Instantaneous reliability of the system at time $\mathrm{t}$

$I A(t)$ : Instantaneous availability of the system at time $\mathrm{t}$

$I M(t)$ : Instantaneous maintainability of the system at time $\mathrm{t}$

\subsection{Instantaneous Reliability Measures}

Instantaneous failure rater $(t)$ of the system at time $t$ is :

$$
r(t)=\frac{f(t)}{1-F(t)}
$$

Instantaneous reliability $I R(t)$ of the system at time $t$ is :

$$
I R(t)=E X P\left(-\int_{0}^{t} r(u) d u\right)
$$

Instantaneous availability $I A(t)$ ofthe system at time $\mathrm{t}$ is:

$$
I A(t)=I R(t)
$$

Instantaneous maintainability $I M(t)$ of the system at time is:

$$
I M(t)=\int_{0}^{t} g(u) d u
$$

Instantaneous repair rate $h(t)$ of the system at time $t$ is:

$$
h(t)=\frac{g(t)}{1-I M(t)} .
$$

\section{Results and Discussions}

\subsection{Example 1 (Supposing the failure and repair rates following the same distribution):}

Assumed that the time to failure and time to repair the system follows Weibull distribution:

$$
f(t)=\lambda \alpha t^{\propto-1} \operatorname{Exp}\left(-\lambda t^{\propto}\right),
$$




$$
g(t)=\beta \theta t^{\theta-1} \operatorname{Exp}\left(-\beta t^{\theta}\right) .
$$

Let the set of parameters be used for this particular example: $\lambda=0.002, \propto=4, \beta=0.006$ and $\theta=6$

Observations made from results obtained for example 1:

1. From table 1 , figure 1 and figure 2 , observed that the instantaneous repair rate $h(t)$ and instantaneous failure rate $\mathrm{r}(\mathrm{t})$ both increases as time $\mathrm{t}$ increases. Furthermore, from figure 3 , observed that, the instantaneous repair rate $\mathrm{h}(\mathrm{t})$ increases as the instantaneous failure rate $\mathrm{r}(\mathrm{t})$ increases .

2. From table 1 and figure 4, observed that, the instantaneous reliability and instantaneous availability decreases as time $t$ increases. While, the instantaneous maintainability increases as time $\mathrm{t}$ increases.

3. Also, observed from table 1 and figure 5, the instantaneous reliability and instantaneous availability decreases as the instantaneous failure rate increases. While, the instantaneous maintainability increases as instantaneous failure rate increases.

4. Also, observed from table 1 and figure 6, the instantaneous reliability and instantaneous availability decreases as instantaneous repair rate increases. While, the instantaneous maintainability increases as instantaneous repair rate increases.

Observations made from 2, 3, and 4 above, showed that maintainability is equivalent to un reliability

Table 1. Results obtained from the evaluation of instantaneous reliability measures when the failure rate and repair rate both followed Weibull distribution

\begin{tabular}{|c|c|c|c|c|c|}
\hline $\mathbf{t}$ & $\mathbf{r}(\mathbf{t})$ & $\mathbf{h}(\mathbf{t})$ & $\mathbf{I A}(\mathbf{t})$ & $\mathbf{I R}(\mathbf{t})$ & $\mathbf{I M}(\mathbf{t})$ \\
\hline 1 & 0.080 & 0.036 & 0.994018 & 0.994018 & 0.001998 \\
\hline 2 & 0.640 & 2.304 & 0.953134 & 0.953134 & 0.015873 \\
\hline 3 & 2.160 & 26.244 & 0.850441 & 0.850441 & 0.052568 \\
\hline 4 & 5.120 & 147.45 & 0.681131 & 0.681131 & 0.120147 \\
\hline 5 & 10.00 & 562.50 & 0.472367 & 0.472367 & 0.221199 \\
\hline 6 & 17.28 & 1679.62 & 0.273624 & 0.273624 & 0.350791 \\
\hline 7 & 27.44 & 4235.36 & 0.127709 & 0.127709 & 0.496414 \\
\hline 8 & 40.96 & 9437.18 & 0.046328 & 0.046328 & 0.640845 \\
\hline 9 & 58.32 & 19131.88 & 0.012601 & 0.012601 & 0.767299 \\
\hline 10 & 80.00 & 36000.00 & 0.002479 & 0.002479 & 0.864665 \\
\hline
\end{tabular}

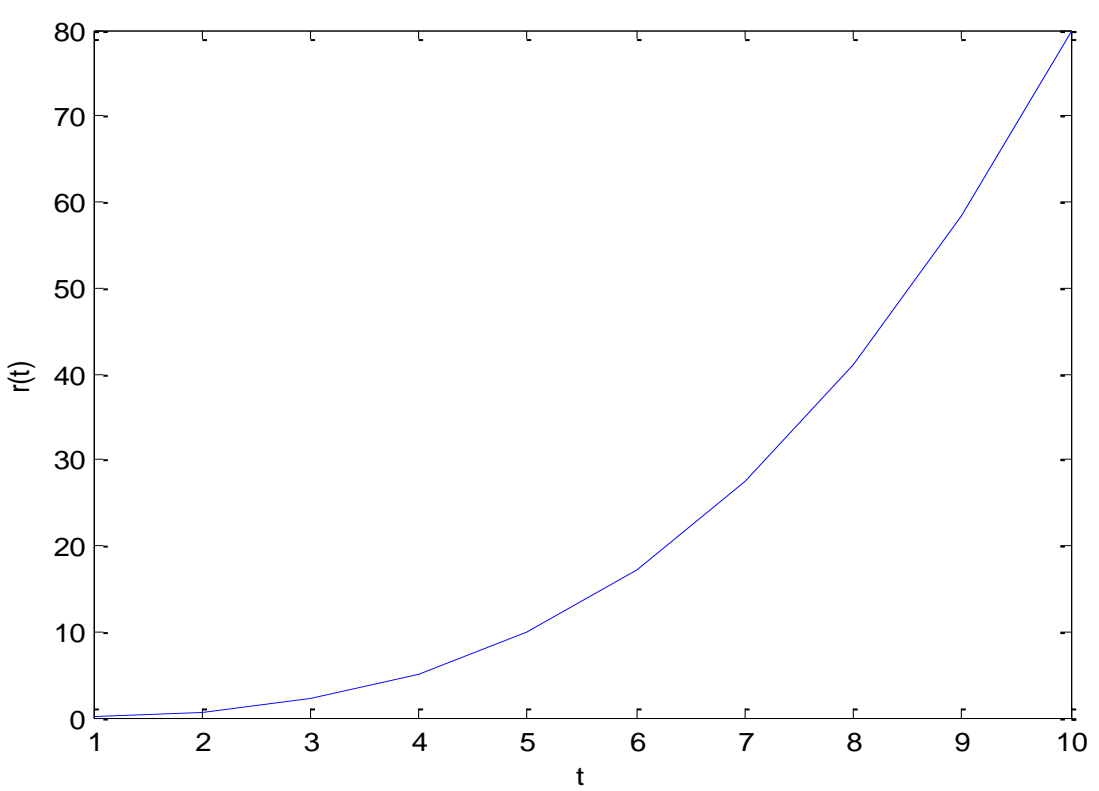

Figure 1. The plot of instantaneous failure rate $\mathbf{r}(\mathbf{t})$ against time $t$. 


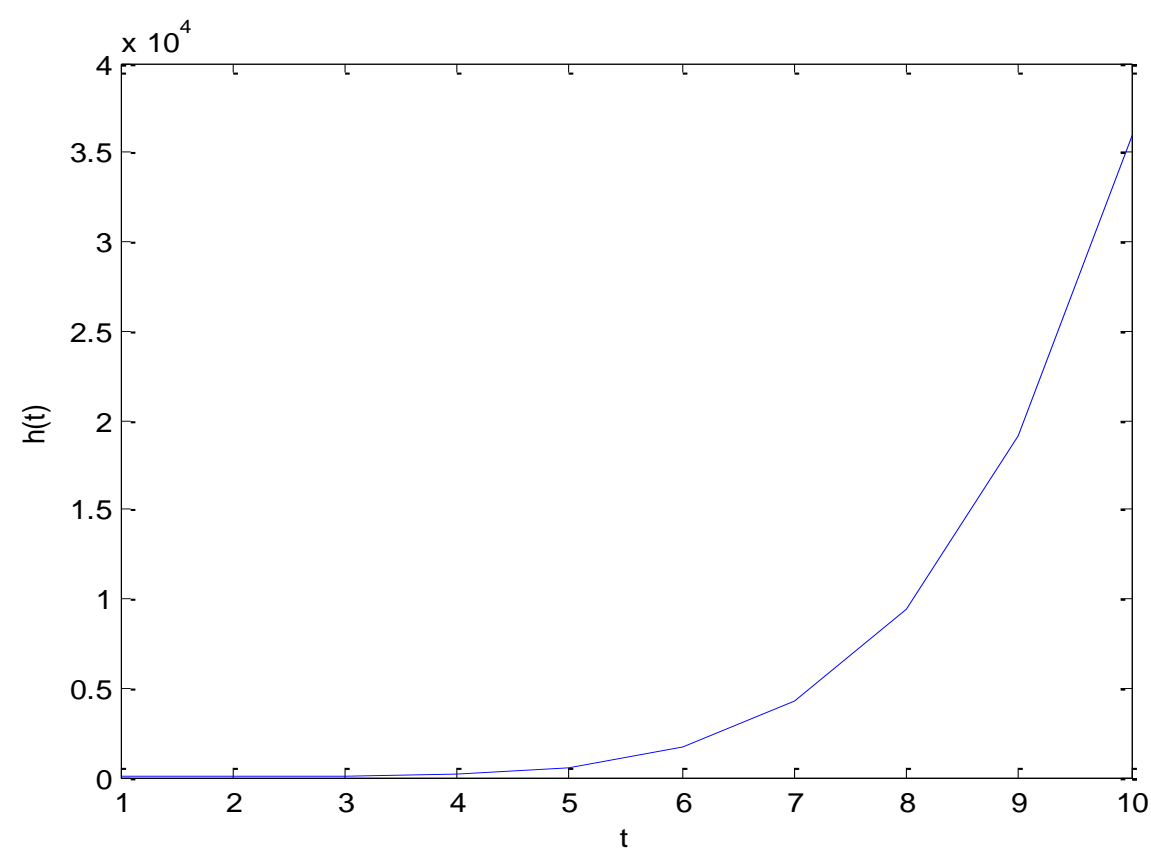

Figure 2. The plot of instantaneous repair rate $h(t)$ against time $t$.

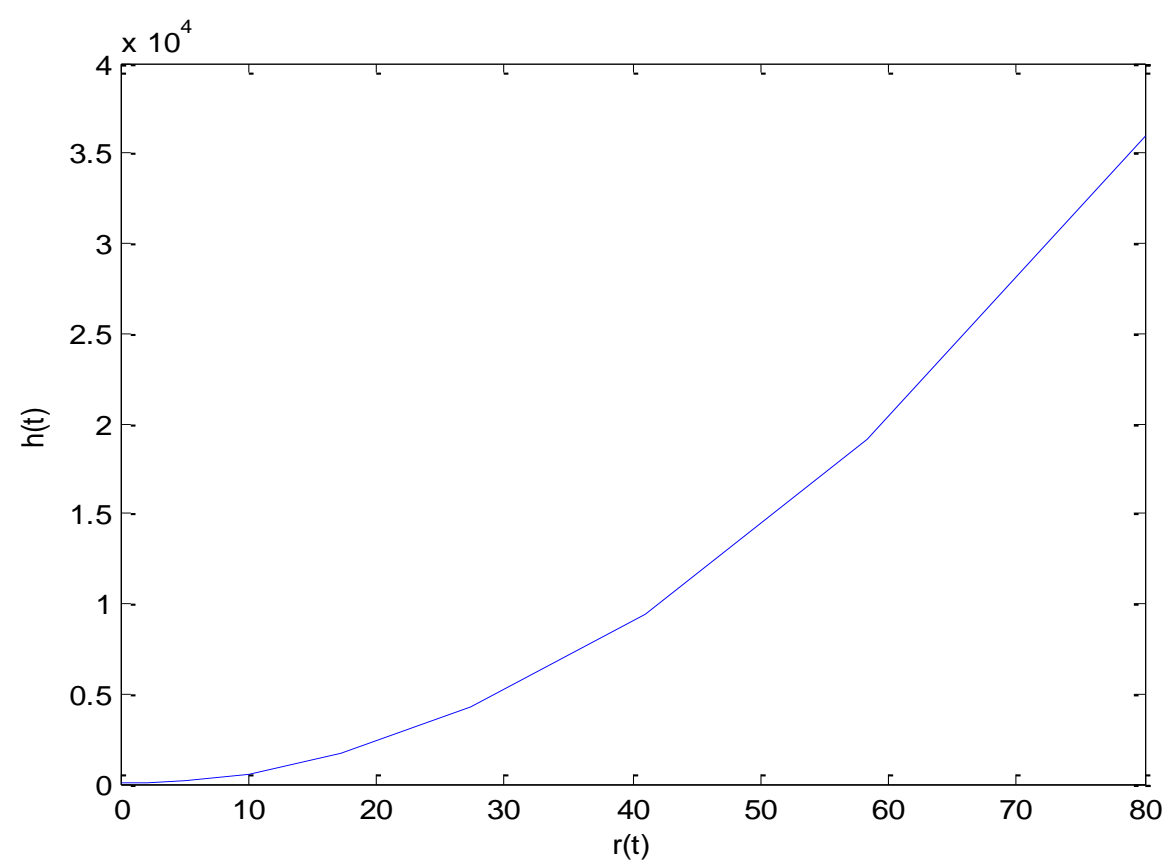

Figure 3. The plot of instantaneous repair rate $h(t)$ against instantaneous failure rate $r(t)$.

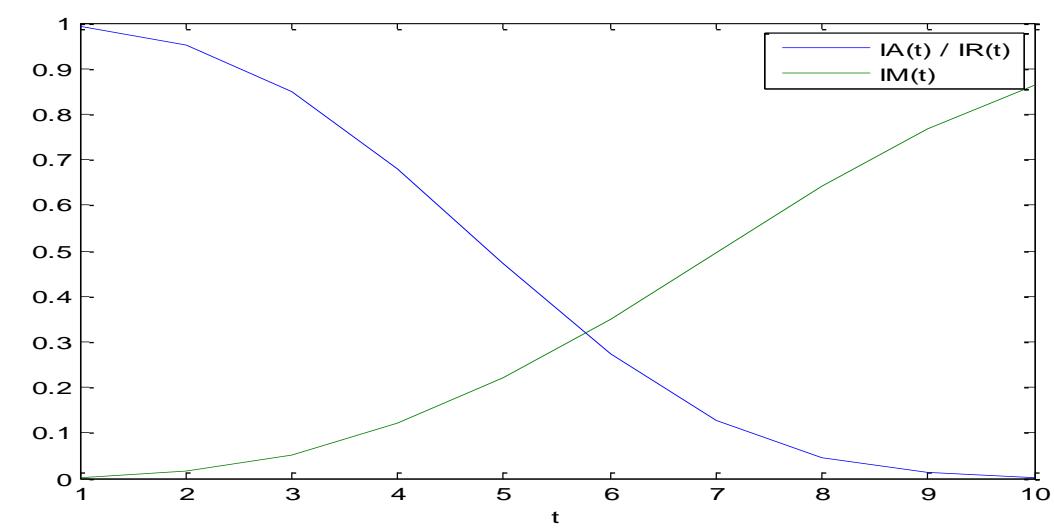

Figure 4.The plot of instantaneous reliability/availability and instantaneous maintainability against time $\mathbf{t}$ 


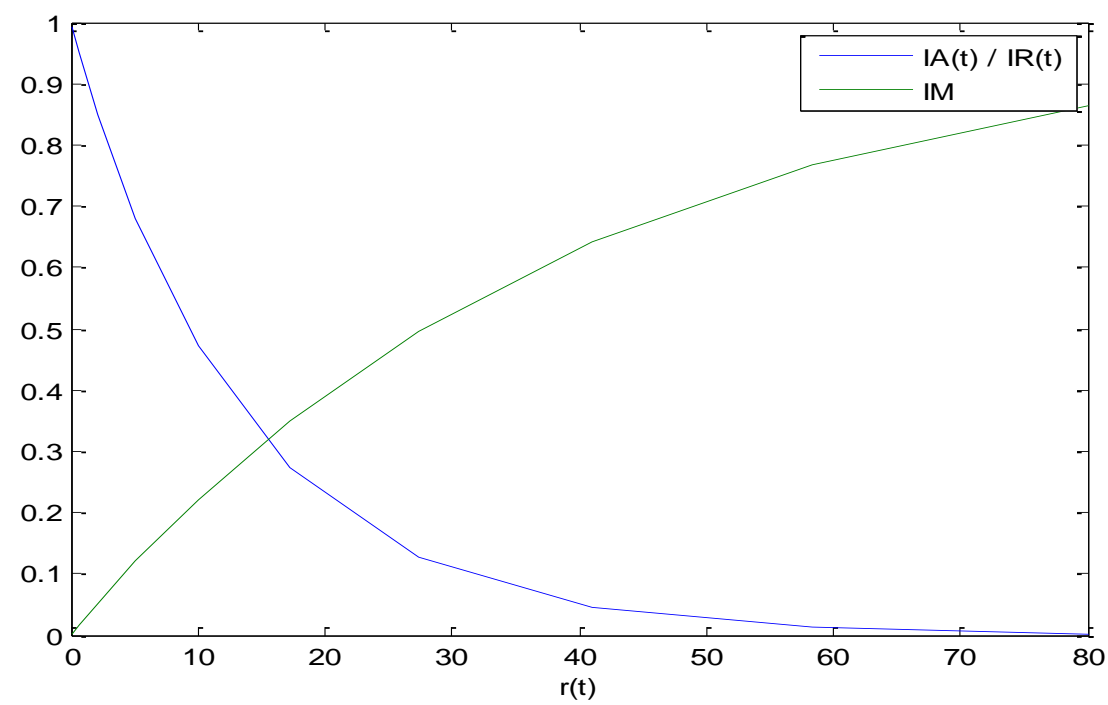

Figure 5. The plot of instantaneous reliability/availability and instantaneous maintainability against instantaneous failure rate $\mathbf{r}(\mathbf{t})$

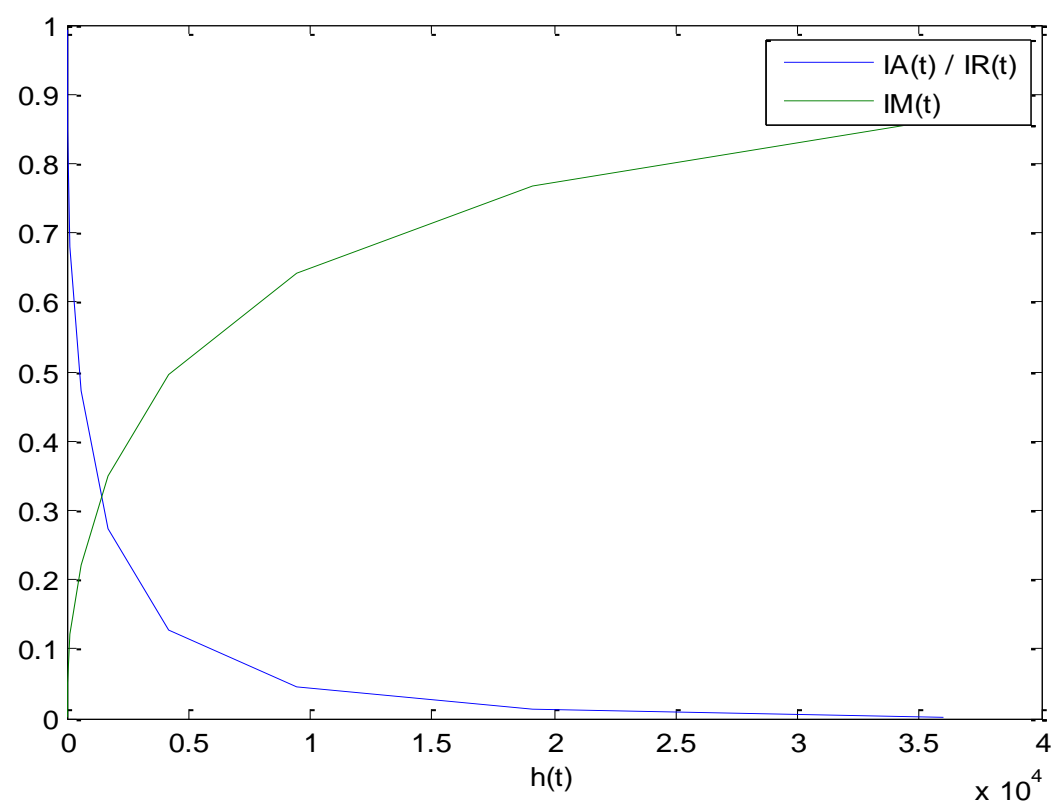

Figure 6. The plot of instantaneous reliability/availability and instantaneous maintainability against instantaneous repair rate $h(t)$

\subsection{Example 2 (failure and repair rates follows different distributions):}

Assumed that the time to failure of the system follows Weibull distribution:

$$
f(t)=\lambda \alpha t^{\propto-1} \operatorname{Exp}\left(-\lambda t^{\propto}\right) .
$$

While the repair time of the system follows power law model:

$$
g(t)=\beta \theta(\beta t)^{\theta-1} \operatorname{Exp}\left(-(\beta t)^{\theta}\right) .
$$

Let the set of parameters be used for this particular example: $\lambda=0.01, \propto=3, \beta=0.01$ and $\theta=3$. 
Table 2. Results obtained for evaluating instantaneous reliability measures when the failure rate follows Weibull distribution and the repair rate follows power law

\begin{tabular}{|c|c|c|c|c|c|}
\hline $\mathbf{t}$ & $\mathbf{r}(\mathbf{t})$ & $\mathbf{h}(\mathbf{t})$ & $\mathbf{I A}(\mathbf{t})$ & $\mathbf{I R}(\mathbf{t})$ & $\mathbf{I M ( t )}$ \\
\hline 1 & 0.03 & 0.003 & 0.990050 & 0.990050 & 0.001000 \\
\hline 2 & 0.12 & 0.012 & 0.923116 & 0.923116 & 0.007968 \\
\hline 3 & 0.27 & 0.027 & 0.763379 & 0.763379 & 0.026639 \\
\hline 4 & 0.48 & 0.048 & 0.527292 & 0.527292 & 0.061995 \\
\hline 5 & 0.75 & 0.075 & 0.286505 & 0.286505 & 0.117503 \\
\hline 6 & 1.08 & 0.108 & 0.115325 & 0.115325 & 0.194265 \\
\hline 7 & 1.47 & 0.147 & 0.032387 & 0.032387 & 0.290362 \\
\hline 8 & 1.92 & 0.192 & 0.005976 & 0.005976 & 0.400704 \\
\hline 9 & 2.43 & 0.243 & 0.000682 & 0.000682 & 0.517609 \\
\hline 10 & 3.00 & 0.300 & $4.54 \mathrm{E}-05$ & $4.54 \mathrm{E}-05$ & 0.632121 \\
\hline
\end{tabular}

Observations made from results obtained for example 2:

1. From table 2, figure 7 and figure 8 , observed that the instantaneous repair rate $h(t)$ and instantaneous failure rate $r(t)$ both increases as time $t$ increases. Furthermore, from figure 10, observed that, the instantaneous repair rate $\mathrm{h}(\mathrm{t})$ increases as the instantaneous failure rate $\mathrm{r}(\mathrm{t})$ increases .

2. From table 2 and figure 9, observed that, the instantaneous reliability and instantaneous availability decreases as time $t$ increases. While, the instantaneous maintainability increases as time $\mathrm{t}$ increases.

3. Also, observed from table 2 and figure 11, the instantaneous reliability and instantaneous availability decreases as the instantaneous failure rate increases. While, the instantaneous maintainability increases as instantaneous failure rate increases.

4. Also, observed from table 2 and figure 12, the instantaneous reliability and instantaneous availability decreases as instantaneous repair rate increases. While, the instantaneous maintainability increases as instantaneous repair rate increases.

5. Observations made from 2,3 , and 4 above, showed that maintainability is equivalent to un reliability

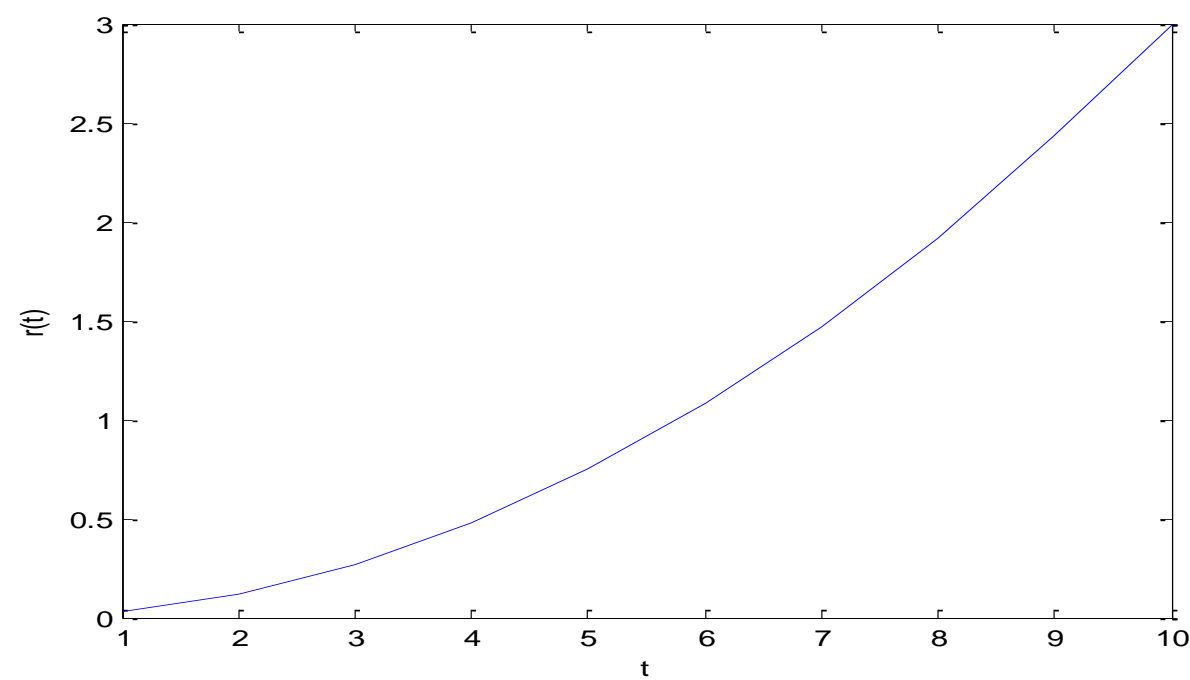

Figure 7.The plot of instantaneous failure rate $r(t)$ against time $t$ 


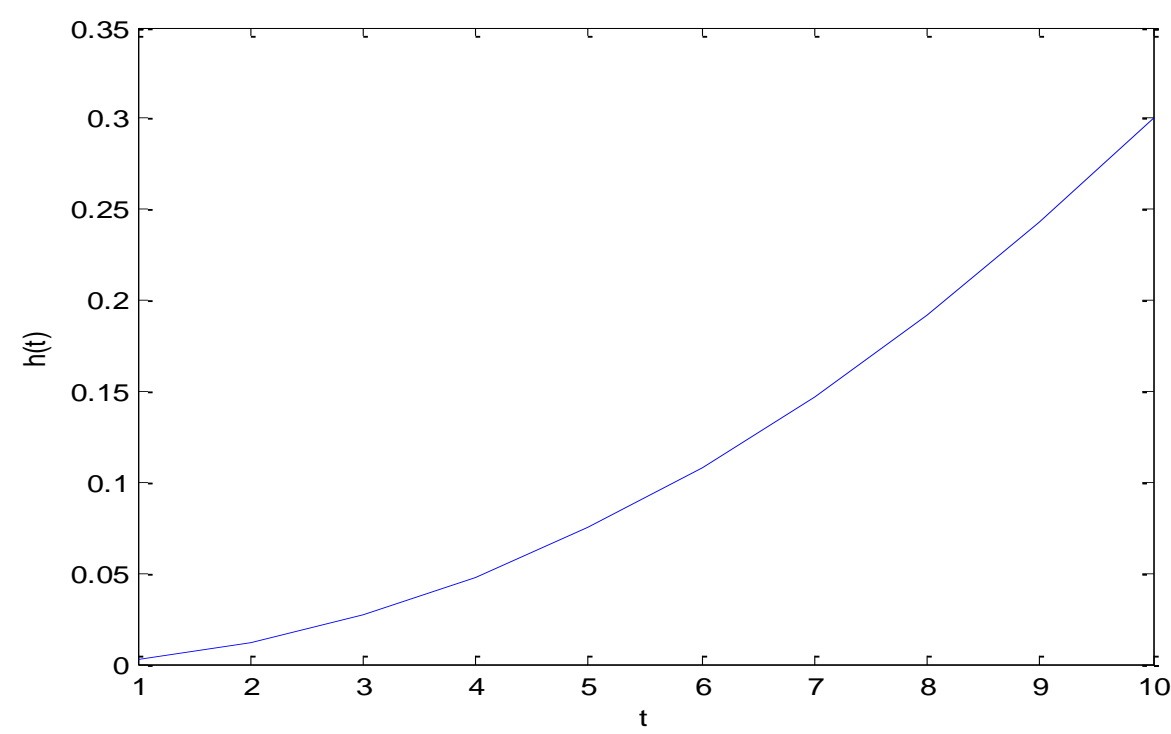

Figure 8. The plot of instantaneous repair rate $h(t)$ against time $t$

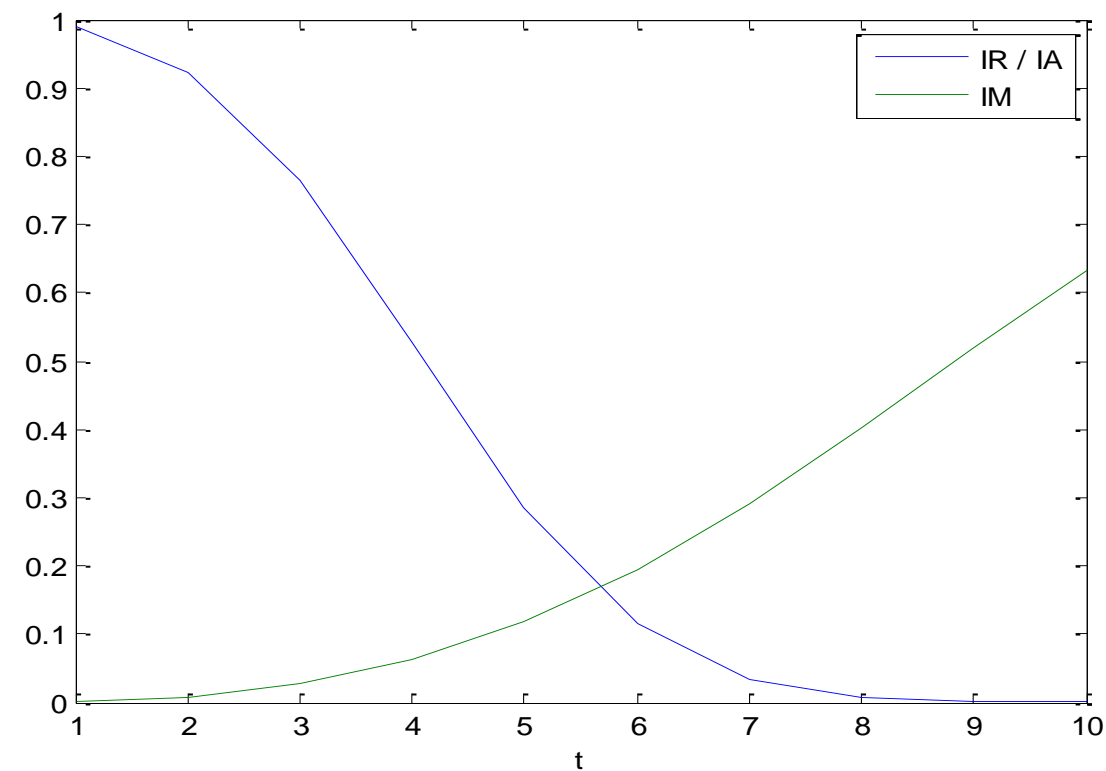

Figure 9.The plot of instantaneous avail./ relaib. and instantaneous maintainability against time $t$

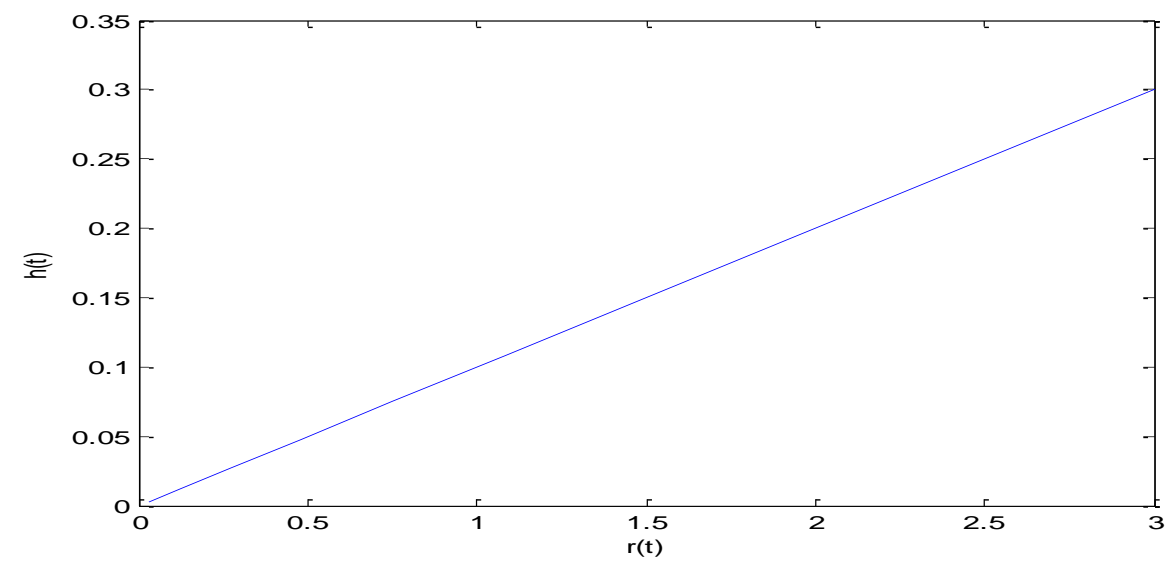

Figure 10. The plot of instantaneous repair rate against instantaneous failure rate 


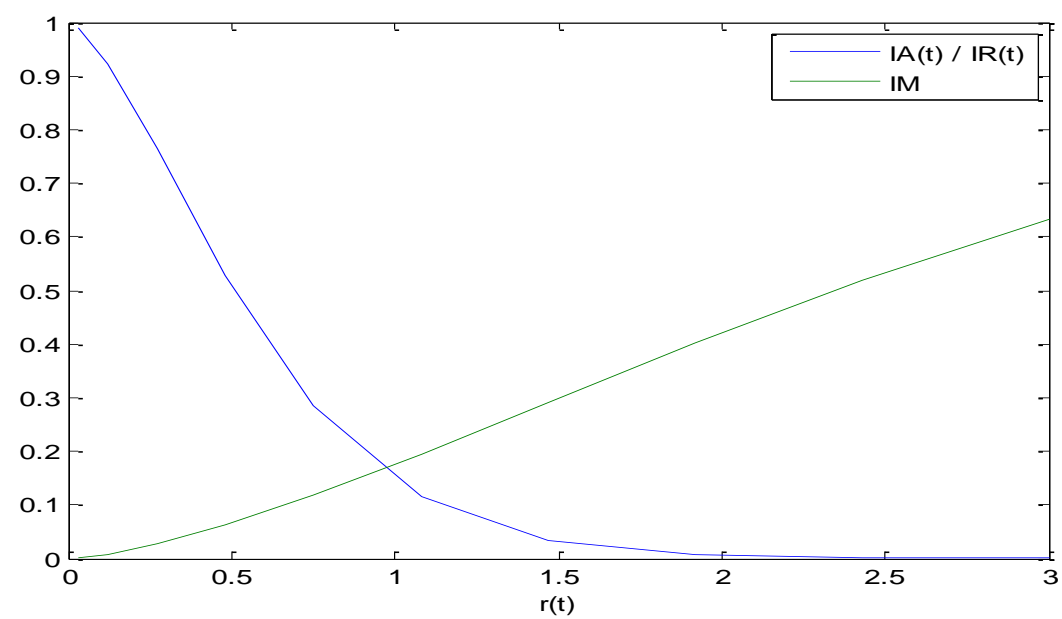

Figure 11. The plot of instantaneous avail./reliab. and instantaneous against instantaneous failure rate

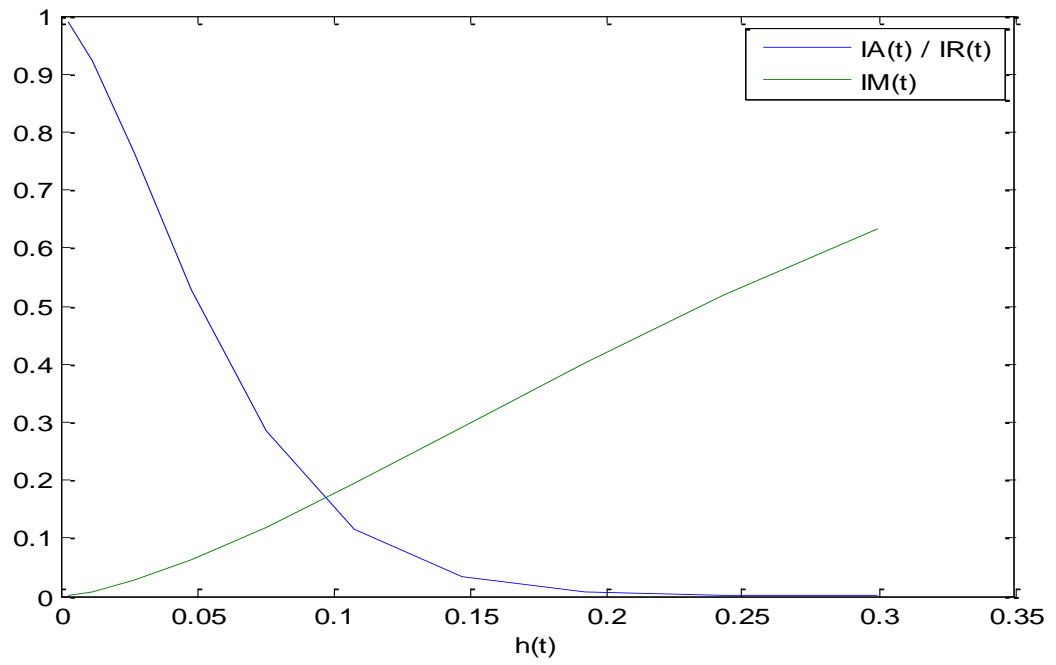

Figure 12. The plot of instantaneous availability / reliability and instantaneous maintainability against instantaneous repair rate

\section{Conclusion}

In attempting to understand the system behavior, the characteristics of instantaneous reliability measures (which are: instantaneous failure rate, instantaneous repair rate, instantaneous maintainability, instantaneous reliability and instantaneous availability) with time $t$ and their relationships are need to be fully investigated. Which provide full information on deterioratingsystems, and make decisions on which type of maintenance policy need to be applied in maintaining the systems. Because systems, have different pattern and manner of deteriorating.

\section{References}

[1] S. M.Aliyu, I. Yusuf ,U.A. Ali.,2015. Availability and Profit Optimization of Series- Parallel System Consisting with Linear Consecutive Cold Standby Units. Journal of Applied Mathematics 6,332- 344.

[2] R.E.Barlow , F.Proschan , 1965. Mathematical Theory of Reliability. Wiley, New York

[3] E. Elsayed, , 1996. Reliability Engineering, Addison Wesley, Reading, MA.

[4] T. Nakagawa, 2005.Maintenance Theory of Reliability. Springer-Verlag, London Limited.

[5] H. Pham, 2003. Handbook of Engineering. Springer- Verlag, London Limited. 
[6] K. Suleiman, U. Ali, I. Yusuf , 2013. Assessment of Reliability and Availability of SeriesParallel Subsystems. 3(9), 2224- 5804.

[7] Sun et al., 2008. Reliability Modeling and Analysis of Series- Parallel Hybrid Multi Operational Manufacturing System Considering Dimensional Quality, Tool Degradation and System Configuration. International Journal of Production Economics, 114, 149-164.

[8] H. Wang, and H. Pham, (2006). Reliability and Optimal Maintenance. Springer-Verlag London Limited.

[9] I. Yusuf, N. Hussaini, 2012. Evaluation of Reliability and Availability Characteristics of 2- out3 Standby System Under a Perfect Repair Condition. American Journal of Mathematics. 2(5),114119.

[10]B. Yusuf, Yusuf, I.,2013. Evaluation Some Reliability Characteristics of a System Under Three Types of Failures with Repair- Replacement at Failure. American Journal of Operational Research, 3(3), 83-91.

[11] I.Yusuf, N. Hussaini, B. M.Yakasai, 2014. Some Reliability Measures of a Deteriorating System. International Journal of Applied Mathematical Research, 3(1), 23-29. 